\title{
Evaluating the effectiveness of a multivariate autoregressive model in predicting the time course of dynamic facial behaviours
}

\author{
David M. Watson*, Alan Johnston \\ School of Psychology, University of Nottingham, Nottingham, UK, NG7 2RD \\ * Corresponding author: david.watson@nottingham.ac.uk
}

\begin{abstract}
Faces convey critical information about people, such as cues to their identity and emotional state. In the real world, facial behaviours evolve dynamically and encapsulate a range of biological motion signals. Furthermore, behavioural and neuroimaging studies have demonstrated that human observers are sensitive to this temporal information. The presence of systematic temporal changes in the face implies the possibility of predicting the evolution of dynamic facial behaviours. We video recorded subjects delivering positive or negative phrases, and used a PCA-based active appearance model to capture critical dimensions of facial variation over time. We applied multivariate autoregressive models to predict PCA scores of future frames from the frames immediately preceding them, up to a lag of $200 \mathrm{~ms}$ prior to the target frame. These models did successfully predict future frames, but they did not benefit from extending the temporal support, suggesting they relied primarily on image similarity between consecutive frames. We next used hidden Markov models to segment videos into shorter sequences comprising more consistent facial behaviours. The Markov models successfully extracted distinct facial basis states, however segmenting the data by state did not yield any predictive benefit to autoregressive models fit within those states. We conclude that autoregressive models have only limited predictive power in the context of facial expression analysis.
\end{abstract}

Keywords: face perception; dynamic faces; ambient faces; computational neuroscience; autoregressive models. 


\section{Introduction}

Human faces convey an abundance of information about a person. For instance, they carry critical cues to a person's identity (Ellis, 1975), social status (Oosterhof \& Todorov, 2008), speech signals (Rosenblum \& Saldaña, 1996; Rosenblum et al., 1996), and emotional state (Bruce \& Young, 1986). The perceptual system can extract these features despite substantial facial variability both between and within people, for instance due to changes in the environment or viewpoint (Jenkins et al., 2011).

In the real world, almost all the faces we encounter are dynamic, with both internal and external features in a constant state of flux. The human perceptual system is able to capitalise on the dynamic nature of faces, extracting key biological motion signals to support perception of those faces. For instance, behavioural evidence suggests a processing advantage for dynamic over static faces when distinguishing between both facial identities (Knight \& Johnston, 1997; Pike et al., 1997; Lander et al., 1999; Lander \& Chuang, 2005) and emotional expressions (Bould \& Morris, 2008; Trautmann et al., 2009), especially under challenging viewing conditions (Lander \& Butcher, 2015). Furthermore, neurological models of face processing networks support the role of a dorsal pathway specialised for the extraction of dynamic facial features (O’Toole et al., 2002; O’Toole \& Roark, 2010; Bernstein \& Yovel, 2015). Indeed, multiple face-selective patches in the superior temporal sulcus display preferential activation for dynamic over static facial stimuli (Schultz \& Pilz, 2009; Trautmann et al., 2009; Pitcher et al., 2011; Furl et al., 2015; Sliwinska et al., 2020), with some more anterior patches only responding selectively to dynamic faces (Zhang et al., 2020). Biological motion of nonface stimuli can also represent key personal information; for instance body posture and gait can convey a person's emotional state (Roether et al., 2009).

Despite evidence for the importance of dynamic information in face perception, much research to date has employed static images of faces taken under tightly controlled experimental conditions. Such approaches aim to control extraneous confounds between stimuli, but in doing so also risk controlling away diagnostic features of the stimuli such as within-person variability (Jenkins et al., 2011). Similarly, many computational models of face perception are trained on static rather than dynamic face stimuli (Calder et al., 2001; Cao et al., 2018). In a recent study (Watson et al., 2020), we proposed a computational approach to address previous limitations. We recorded videos of people displaying dynamic and natural 
facial behaviours while reading short sentences conveying either good or bad news. Importantly, subjects were not asked to deliberately or artificially pose any specific facial expressions. We used a two-frame version of the Multi-channel Gradient Model (McGM; Johnston et al., 1992, 1999; Cowe, 2003) to measure form and texture changes over time, and a Principal Components Analysis (PCA; Turk and Pentland, 1991) then captured the critical dimensions of this variation. Using a machine learning approach we could both discriminate and reconstruct the emotional valence of the expressions, demonstrating the utility of this approach for representing patterns of dynamic behaviour in natural facial expressions. This approach does capture dynamic motion information, in the sense of representing changes in form and texture between frames, however it does not consider the temporal structure of that information. Rather, each frame is represented as an independent sample and the order of the frames is unimportant to the model. Thus, the model as it stands provides a representation of the ways in which a facial image can change, but it remains unclear whether it is possible to utilise temporal trajectories of change within these representations to predict the dynamic evolution of facial behaviours.

Here we extend the analysis of Watson et al. (2020), with the aim of investigating whether it is possible to predict the time course of dynamic facial behaviours. Specifically, we test whether information about the recent history of a face's behaviours can predict future behaviours of that face. We again employ an McGM-PCA active-appearance model to capture key dimensions of form and texture changes in the face over time. We then employ multi-variate autoregressive (MVAR) models to predict PCA scores of future frames from those of the frames preceding them - up to a lag of $200 \mathrm{~ms}$ prior to the target frame. MVAR models are frequently used for temporal forecasting, and have been widely applied in a multitude of fields including economics (Sims, 1980), meteorology (Richardson, 1981), and neuroscience (Harrison et al., 2003). We additionally employ hidden Markov models (HMMs) to extract underlying behavioural states, in order to identify sequences of more consistent facial behaviours for further MVAR analyses. If autoregressive models are able to capture the time course of facial behaviours, we would expect that past frames will successfully predict future frames, and that prediction accuracy will increase as more past temporal information is included in the model. 


\section{Methods}

\section{Recordings}

The recordings used here have previously been described in Watson et al. (2020). Three subjects ( 2 females, 1 male, age range $26-42$ ) were video recorded. The study was approved by the ethics committee of the School of Psychology at the University of Nottingham (ethics approval number: 717) and conducted in accordance with the guidelines and regulations of this committee and the Declaration of Helsinki. All subjects provided informed written consent to take part in the study and for their likeness to be used in publication.

Recordings were made in an anechoic chamber against a uniform visual background. Videos were acquired on a Sony HXR-NX5U NXCAM camera connected to an Atomos Ninja-2 recorder that recorded videos in Apple ProRes RAW format. Videos were acquired at a resolution of $1920 \times 1080$ pixels and at $25 \mathrm{fps}$ with a $6.67 \mathrm{~ms}$ exposure. Videos were then encoded using MPEG-4 lossless compression prior to further processing.

Each subject delivered a total of 20 unique phrases to camera that conveyed either good news (e.g. "Good news, you've got the job!") or bad news (e.g. "I'm sorry to say, the operation didn't go well"). A full list of the phrases is provided in Table S1. Subjects 1 and 2 delivered 15 repeats of each phrase (300 total), and Subject 3 delivered 16 repeats (320 total). Subjects were instructed to deliver the phrases in whatever manner felt most natural to them: they were not required to deliberately pose or perform any specific expressions or actions. To assist subjects, they viewed pre-recorded silent videos of various recipients on a teleprompter placed directly in front of the camera. These videos showed video-conference style calls obtained from YouTube and helped give subjects the impression of a person listening to them while they delivered their phrases.

Phrase segments were clipped from the recordings following a semi-automated procedure. The Google Cloud Speech-to-Text algorithm (https://cloud.google.com/speech-to-text) was used to generate timestamps for each word within each clip. Clips were then cut to the timestamps of the first word's onset and final word's offset, plus an extra 200ms (5 frames) before and 400ms (10 frames) after to ensure facial movements starting before and ending after the vocalisations were also included. Manual corrections were applied where necessary. 
The average and standard deviation of durations across clips were $3.34 \pm 0.61 \mathrm{~s}, 3.17 \pm 0.48 \mathrm{~s}$, and $3.28 \pm 0.54 \mathrm{~s}$ for each of the three subjects respectively.

\section{MCGM-PCA model}

A two-frame version of the Multi-channel Gradient Model (McGM; Johnston et al., 1992, 1999; Cowe, 2003) was used to capture and register the textures in each frame to a reference image. First, for each clip a Haar cascade face-detection algorithm in OpenCV (https://opencv.org/) was used to establish the average position of the face within the frame, and the video was then cropped to a square bounding box centred around this position. This ensured the face was located approximately centrally within the scene. Each frame was then downsampled to a resolution of $128 \times 128$ pixels using an anti-aliasing filter.

Cropped and downsampled clips were entered into the McGM (Figure 1a). For each frame, a set of warp components were calculated to register the textures in that frame onto a standard reference image. The reference was initially set as an individual frame selected from one of the recording sequences. However, to provide a more standardised reference, the original reference was then replaced with the average of all textures after warping. This process was then repeated three times, re-calculating the warps and updating the reference with the average warped texture each time, to allow the reference to stabilise. The McGM yields a 5-channel image for each input frame, comprising the $x$ - and $y$-direction warp components and a "shape-free" version of the RGB textures warped to the final average reference. Flattening these images to vectors allows representing each frame as a separate sample within an 81,920 -dimensional $(128 \times 128 \times 5)$ feature space. Note that the two-frame version of the McGM used here for the purpose of image registration (Cowe, 2003) differs from some previous applications of the model that instead computed local image velocities over extended temporal sequences (Johnston et al., 1992, 1999).

From this point onwards, samples from each of the two phrase-types - "Good news..." (GN) and "I'm sorry to say..." (ISTS) - were processed separately. In addition, a split-half crossvalidation scheme was employed, such that half of the clips were allocated to one split, and the remaining half to the other split (giving 5 unique phrases per phrase-type within each split). This allowed the models to be fit to data from clips in one data split, then validated on independent data from the held-out clips in the other split. The data were split according to 
the even/odd numbering of the clips to mitigate systematic changes that might correlate with the recording order (i.e. even numbered clips were allocated to one split, and odd-numbered clips to the other split).

a

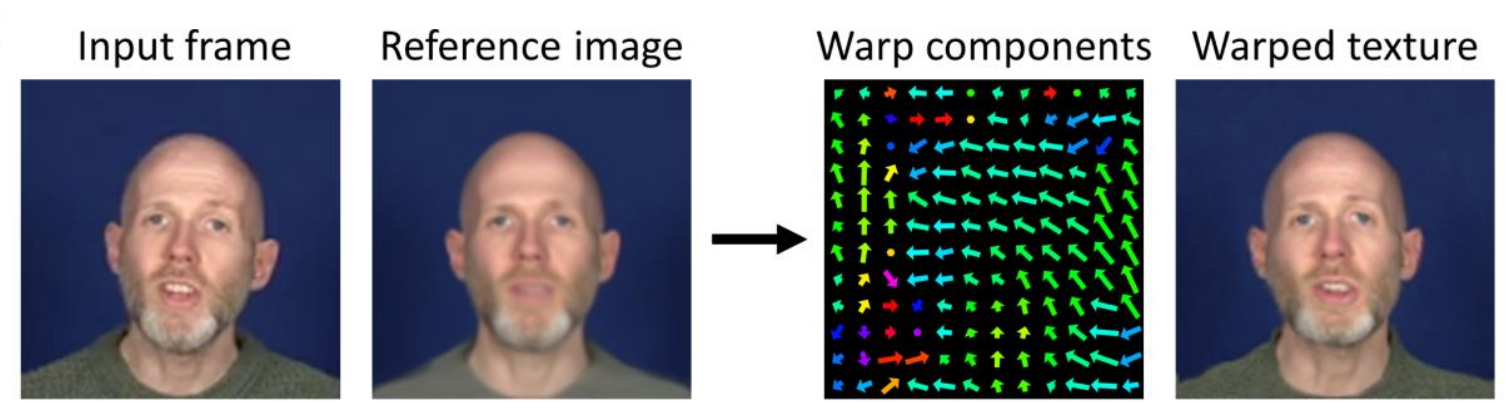

b

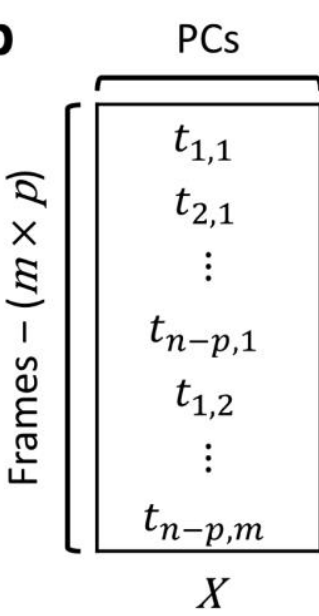

(Single-lag)

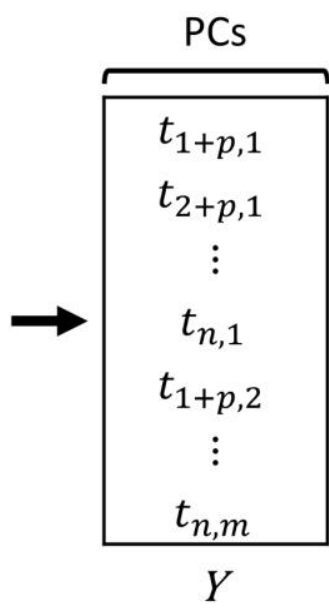

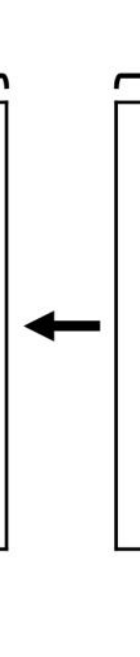

$p \times \mathrm{PCS}$

\begin{tabular}{|cccc|}
\hline$t_{1,1}$ & $t_{2,1}$ & $\cdots$ & $t_{p, 1}$ \\
$t_{2,1}$ & $t_{3,1}$ & $\cdots$ & $t_{1+p, 1}$ \\
$\vdots$ & $\vdots$ & $\ddots$ & $\vdots$ \\
$t_{n-p, 1}$ & $t_{1+n-p, 1}$ & $\cdots$ & $t_{n-1,1}$ \\
$t_{1,2}$ & $t_{2,2}$ & $\cdots$ & $t_{p, 2}$ \\
$\vdots$ & $\vdots$ & $\ddots$ & $\vdots$ \\
$t_{n-p, m}$ & $t_{1+n-p, m}$ & $\cdots$ & $t_{n-1, m}$ \\
\hline
\end{tabular}

$X$

(Multi-lag)

Figure 1. (a) McGM procedure. Each frame is registered to a standard reference image, such that it is represented by a 5 -channel image comprising the $x$ - and $y$-warp components and RGB warped textures. Vectorising these images allows representing each frame as a sample within a high-dimensional feature space, which is then reduced via PCA. Samples are contained in an $N$-by- $d$ matrix, where $N$ is the total number of frames over clips, and $d$ the number of principal components. (b) MVAR procedure. The $d$ length vector $t_{i, j}$ represents the PCA scores for the $i^{\text {th }}$ frame of the $j^{\text {th }}$ clip, up to $n$ frames per each of $m$ clips. Scores for each clip are split into leading and trailing sequences, separated by a lag of $p$ frames. Scores within each set are then recombined by vertically stacking across clips, yielding $(N-m \times p)$ samples per set. In the single-lag approach, leading sets comprise a single sequence from each clip: the first frame up to $p$ frames before the final frame. In the multi-lag approach, leading sets comprise multiple sequences from each clip: starting with the sequence comprising the first frame up to $p$ frames before the final frame, then offset by one frame each time up to the final sequence comprising the $p^{\text {th }}$ frame up to the penultimate frame. PCA scores are then horizontally stacked across each sequence, yielding a $(p \times d)$ length vector for each sample. In both cases, a multivariate regression model is used to map scores from the leading-frames array $(X)$ to the trailing-frames array $(Y)$. 
The dimensionality of the McGM space was reduced via a principal components analysis (PCA; Turk and Pentland, 1991; Calder et al., 2001). This was performed both on the full datasets, and within each fold of the split-half cross-validated datasets. Each PCA thus included frames/samples from 150 clips (160 clips for Subject 3) per phrase-type for the full datasets, or from 75 clips (80 clips for Subject 3) per phrase-type per split for the cross-validated datasets. A sufficient number of components were retained to explain $75 \%$ of the variance across samples. The numbers of principal components retained for each subject, phrase type, and cross-validation fold are shown in Table 1. When operating on the cross-validated datasets, each PCA was fit to data within its training cross-validation fold (either odd or even clips), and then applied to the data in both folds. In this way, for each PCA both training and testing data were brought into the same space, but the derivation of the PCA coefficients was based solely on the training data. Successful projection of the testing data into the PCA space thus requires generalisation of the data between cross-validation folds. Finally, PCA scores were normalised by z-scoring values along each principal component within each clip independently. This was performed to standardise the variance of scores along each principal component whilst also removing global trends between the clips.

Table 1. Number of principal components retained to explain $75 \%$ of variance over samples. Values given for each subject, phrase-type (GN = "Good news", ISTS = "I'm sorry to say"), and for full datasets and each fold of the split-half cross-validated datasets.

\begin{tabular}{ccccc}
\hline Subject & Phrase Type & Full & $\begin{array}{c}\text { Dataset } \\
\text { Even clips }\end{array}$ & Odd clips \\
\hline S1 & GN & 52 & 48 & 46 \\
& ISTS & 52 & 47 & 46 \\
S2 & GN & 14 & 14 & 13 \\
& ISTS & 13 & 11 & 13 \\
S3 & GN & 60 & 53 & 54 \\
& ISTS & 59 & 54 & 54 \\
\hline
\end{tabular}

\section{Multivariate autoregressive model}

We used cross-validated multivariate autoregressive (MVAR) models (Harrison et al., 2003; Hytti et al., 2006) to predict the time course of the facial behaviours by using PCA scores from previous frames to predict scores in future frames (Figure $1 b$ ). Let $n$ be a vector giving the 
number of frames/samples within each clip up to a total of $m$ clips per phrase-type per crossvalidation fold; $n=\left\{n_{1}, n_{2}, \ldots, n_{m}\right\}$. The number of clips $(m)$ equals 75 for Subjects 1 and 2 , and 80 for Subject 3 . The total number of frames/samples $(N)$ is given by the sum over the vector $n$.

The normalised PCA scores for a given phrase-type and cross-validation fold are represented in an $N$-by- $d$ matrix, where $d$ is the number of principal components retained. The $d$ length row vector $t_{i, j}$ within this matrix represents the PCA scores for the $i^{\text {th }}$ frame of the $j^{\text {th }}$ clip. We then vertically slice chunks out of this matrix comprising leading (predictor) and trailing (outcome) frame sequences from each clip, offset by a given lag $p$ (in frames): from a minimum lag of $p=1$ (40ms) up to maximum of $p=5$ (200ms).

We employ two strategies for applying these offsets, differing in how the leading (predictor) frames are collated. In the single-lag approach we select a single leading sequence from each clip, running from the first frame up to $p$ frames before the final frame of the clip. For a given clip $j$, this yields the matrix:

$$
x_{\text {single }}=\left[\begin{array}{c}
t_{1, j} \\
t_{2, j} \\
\vdots \\
t_{n-p, j}
\end{array}\right]
$$

In the multi-lag approach, we select multiple leading sequences from each clip. We again start with the sequence from the first frame up to $p$ frames before the final frame in the clip. We then select further sequences by incrementing the starting and ending frames by one each time, up to the sequence starting on the $p^{\text {th }}$ and ending on the penultimate frame. The parameter $p$ thus determines the order of the model, giving both the maximal offset and the number of lags included in the leading set. Leading sequences are then stacked horizontally. For a given clip $j$, this yields the matrix:

$$
x_{\text {multi }}=\left[\begin{array}{cccc}
t_{1, j} & t_{2, j} & \cdots & t_{p, j} \\
t_{2, j} & t_{3, j} & \cdots & t_{1+p, j} \\
\vdots & \vdots & \ddots & \vdots \\
t_{n-p, j} & t_{1+n-p, j} & \cdots & t_{n-1, j}
\end{array}\right]
$$


The trailing sequences are always selected from the $(1+p)^{\text {th }}$ frame up to the final frame within each clip. For a given clip $j$, this yields the matrix:

$$
y=\left[\begin{array}{c}
t_{1+p, j} \\
t_{2+p, j} \\
\vdots \\
t_{n, j}
\end{array}\right]
$$

Leading and trailing sequences from each clip are then vertically stacked back together over clips to create new matrices with $(N-m \times p)$ rows (total frames minus the frames removed from each clip). The trailing $(Y)$ and single-lag leading matrices $\left(X_{\text {single }}\right)$ each comprise $d$ columns, while the multi-lag leading matrix $\left(X_{\text {multi }}\right)$ comprises $(p \times d)$ columns. Note that the first-order MVAR models $(p=1)$ are equivalent between the single- and multi-lag approaches.

As an example, consider a hypothetical clip comprising 15 frames (all real clips were longer than this) and applying the maximal offset of $p=5$. This would yield leading and trailing sequences each comprising 15 - $5=10$ frames. The trailing sequence would comprise each of the $d$-length PCA score vectors corresponding to frames 6 through 15 inclusive. In the single-lag approach, the leading sequence would comprise each of the $d$-length vectors corresponding to frames 1 through 10 inclusive. In the multi-lag approach, five separate leading sequences of $d$-length vectors would be taken, starting with the sequence comprising frames 1 through 10 inclusive (as per the single-lag approach), but then also the sequences comprising frames 2 through 11, then 3 through 12, then 4 through 13, and finally 5 through 14. The five matrices comprising each of these 10-frame sequences would then be stacked horizontally, yielding a final matrix with 10 rows (frames) and $5 \times d$ columns. This whole process would then be repeated for each clip in turn, and the resulting matrices vertically stacked together over clips.

We next fit a multivariate regression model to map the PCA scores from the leading (predictor) to the trailing (outcome) sequences. Each data matrix was first centred by subtracting the mean over columns - this removes mean trends over components (which don't vary over time) and negates the need for an intercept term in the model. The ordinary least squares solution yields the regression coefficients matrix $B$, with dimensions $d$-by- $d$ in 
the single-lag approach, or $(p \times d)$-by- $d$ in the multi-lag approach. Note that coefficients are obtained from the training portion of the data only (either odd or even clips):

$$
\mathrm{B}=\left(X_{\text {train }}^{T} X_{\text {train }}\right)^{-1} X_{\text {train }}^{T} Y_{\text {train }}
$$

We then use the PCA scores from the testing data portion to predict trailing (outcome) components from the corresponding leading (predictor) components:

$$
\widehat{Y}_{\text {test }}=X_{\text {test }} \mathrm{B}
$$

Prediction accuracy is assessed by correlating the predicted and actual trailing PCA scores across frames, yielding the vector $r=\left\{r_{1}, r_{2}, \ldots, r_{N-m p}\right\}$, where $r_{i}=\operatorname{corr}\left(Y_{i}, \hat{Y}_{i}\right)$. This whole process is then repeated for the other cross-validation fold, such that all data is ultimately used for training and testing. Successful prediction thus requires the mapping between leading and trailing frames to generalise over cross-validation folds.

\section{Hidden Markov model}

To identify underlying basis states in the facial behaviours over time, we fit unsupervised Gaussian hidden Markov models (HMMs) to the normalised PCA scores. These identify a number of underlying hidden states, each modelled by a multivariate Gaussian within the PCA feature space. States are not observable directly, but can be inferred by their "emissions" evident in changes in the PCA scores over time. HMMs were fit to both the full and crossvalidated datasets, and were implemented using the hmmlearn package in Python (https://github.com/hmmlearn/hmmlearn).

\section{Model selection}

We first chose a number of states to estimate (given by the parameter $k$ ). We fit HMMs for $k=2$ up to $k=10$, for each subject and phrase-type separately, and for both the full dataset (without cross-validation) and for each of the odd/even data splits of the cross-validation. To mitigate the effects of local minima on the optimisation of the HMM parameters, each model was fit from 100 different random starts and we selected the iteration yielding the highest likelihood score. We then employed two measures of the model error to assist in choosing an appropriate value of $k$. First, we calculated Bayesian Information Criterion (BIC) scores (Schwarz, 1978; Pohle et al., 2017) for the full-dataset models. The BIC is given as: $\mathrm{BIC}=k \ln (q)-2 \ln (\hat{L})$, where $q$ is the number of model parameters and $\hat{L}$ is the model 
likelihood. Each HMM estimates $k$ starting probabilities, $k^{2}$ transition probabilities, $k \times d$ means, and $k \times d^{2}$ covariances, giving the total number of components as: $q=k+k^{2}+k d+k d^{2}$. Secondly, we calculated cross-validated likelihood values (Celeux \& Durand, 2008). These were derived by fitting each HMM to the PCA scores from one of the cross-validation splits (odd or even clips), and then estimating the model likelihood for scores in the other split. This process is repeated for both cross-validation folds, and the values average over folds.

We also back-projected the full-dataset state means to the image space to help visualise the facial behaviours captured by each state. Each state mean is a $d$ length vector defined within the normalised PCA space. We first transformed these vectors to the original (un-normalised) PCA space by multiplying each vector by the component standard deviations then adding the component means obtained from the original z-scoring operation (see McGM-PCA model methods). As the normalisation was originally performed for each clip separately, we used an average of the component standard deviations and means over clips. Next, we inverted the PCA to transform the state means back to the McGM space, then further inverted the McGM warp to return to the image space. Finally, the image contrast was enhanced using an unsharp filter. The result is a visualisation of the prototypical facial behaviours embodied by each state.

The full-dataset BICs and cross-validated likelihoods are illustrated in Figure S1, and the back-projected state means are illustrated in Figures S2-S7. While each of these measures can assist in the choice of $k$, the final choice is ultimately subjective. We detail these choices below:

- Subject 1, GN: Selected $k=4$. BIC suggests $k=4$, cross-validated likelihood suggests $k=2$. Including 4 states presents a wider variety of expressions and head positions than with fewer states. Additional states do not add much further novelty.

- Subject 1, ISTS: Selected $k=5$. BIC and cross-validated likelihood suggest $k=5$. Additional states do not show further novelty, so we accept objective suggestions.

- Subject 2, GN: Selected $k=7$. BIC suggests $k=10$, cross-validated likelihood suggests $k=9$. Objective measures tend towards upper limit of values tested, but these do not offer substantial novelty over fewer states. Including 6 states adds in a 
"lip-press" expression, and 7 states includes a further "head-tilt", while additional states do not offer much further novelty.

- Subject 2, ISTS: Selected $k=7$. BIC and cross-validated likelihood suggest $k=10$. Again, objective measures suggest the upper limit of values tested, but these do not offer substantial novelty over fewer states. Including 6 states adds a "lip-press + headturn", and 7 states adds a "lips-apart + head-turn", while further states don't add further novelty.

- Subject 3, GN: Selected $k=6$. BIC suggests $k=6$, cross-validated likelihood suggests $k=5$. Including 5 states adds a "broad smile", while 6 states add a "lips-apart + headraised" pose, so we select the larger of the objective recommendations.

- Subject 3, ISTS: Selected $k=7$. BIC and cross-validated likelihood both suggest $k=5$. Increasing to 6 states adds in a "lip-press", and 7 states adds a "moderate grimace", then further states do not add much further novelty.

\section{Time course of posterior probabilities}

To examine how states vary over time, we extracted posterior probabilities for each state and frame from the full dataset. As clips vary in length, the frame timestamps were converted to a normalised scale such that each clip started at 0 and ended at 1 . Posterior probabilities were then binned by the normalised duration into 50 bins each of 0.02 units duration. Values were then averaged over frames and clips within each bin to generate an average time course of posterior probabilities for each state.

We additionally created animations of the back-projected images by weighting them by the posterior probability time courses. An arbitrary duration of 4.5 seconds was chosen for the animation, corresponding to approximately $75 \%$ playback speed relative to the original clips. The posterior probability time courses were linearly interpolated across 135 timepoints (30 fps $\times 4.5 \mathrm{~s}$ ). The state means vectors were back-projected to the McGM space (see above) and weighted by the interpolated posterior probabilities at each time point, and summed over states. This produced an average McGM vector for each timepoint, which was then warped back to the image space. 


\section{Combined HMM-MVAR analysis}

We next used the HMMs to derive frame sequences for each hidden state, to be entered into a series of MVAR analyses. This was performed within the cross-validation framework: for each subject and phrase-type, an HMM was trained on the normalised PCA scores for one of the cross-validation splits (odd or even clips). The number of states $(k)$ was set for each subject and phrase-type as described above, selecting the same number of states for both cross-validation folds. We then generated posterior probabilities for each sample within both data splits, including the split the HMM was trained and the one it was not. For each state and data split, we selected frame sequences from each clip according to the following criteria:

1) The posterior probability was maximal for that state.

2) The posterior probability for that state was greater than 0.95 .

3) The sequence comprised at least 10 frames (the minimum required for a 5 -frame lag).

This generated a set of PCA scores for each state, organised by the state-sequences instead of the original clips. Each HMM thus generated two sets of state-sequences: one for the data split it was trained on, and one for the data split it wasn't trained on. Conversely then, there were also two independent estimates of state-sequences for each data split: one generated by the HMM trained on that split, and one generated by the HMM trained on the other split. Figure $\$ 8$ shows the proportion of frames allocated to each state.

These sequences were then entered into a series of MVAR analyses. These followed the same procedure as for the standard MVAR analyses described above, but were conducted within each state independently, and operated by splitting leading and trailing sequences within each state-sequence instead of the original clips. The regression model was fit to sequences within the training data portion and validated on sequences within the testing portion, in both cases using the state-sequences generated by the HMM fit to the training data. This generated a vector of correlations measuring the model prediction accuracies for each state, which were then concatenated back together over states. This process was then repeated for the other cross-validation fold. Successful prediction thus requires generalisation over the data splits by both the HMM and MVAR. Note that iterations were skipped where the number of training samples was less than the number of leading (predictor) components, as such cases would yield singular covariance matrices. 


\section{Statistics}

We used a permutation procedure to test the performance of the MVAR models against chance. Frames within leading (predictor) sequences of the training data were randomly reordered within each clip (standard analysis) or state-sequence (HMM analysis) independently. This breaks the temporal correspondence between leading and trailing sequences (note that it is unnecessary to also reorder the trailing sequences). The MVAR model was then fit using the reordered sequences, and validated on the testing data as normal. Note that the correct order of frames is maintained for the testing data, as the aim is to assess the ability of a model trained on permuted data to predict real data. Note also that for multi-lag models the permutation occurs prior to the selection of leading sequences, such that the order of frames across lags within the predictor set is not disrupted (i.e. the order of matrix rows is permuted but not the order of columns). For the standard MVAR analysis we performed 500 permutations per cross-validation fold (1000 total). For the HMM-MVAR analysis we performed 200 permutations per state per cross-validation fold (total permutations varied by the number of states, from 1600 for $k=4$ to 2800 for $k=7$ ).

Each permutation yielded an $(N-m \times p)$ length vector of correlations, which were then concatenated over permutations to generate an empirical null distribution that could be compared against the corresponding true correlation distribution. First, both null and true correlations were converted to units of Fisher's $z$. Next, we calculated the mean of the true distribution, and a corresponding one-tailed $p$-value of observing a mean at least this extreme under the empirical null distribution. The $p$-values were initially defined as the proportion of scores in the empirical null distribution greater than the true mean, then subjected to a Holm-Bonferroni correction for multiple comparisons (Holm, 1979) across the 10 tests ( 2 phrase-types $\times 5$ lags) within each subject.

We also compared the MVAR performance between conditions. Model correlations were converted to Fisher's $z$ and averaged over samples. The mean correlations for each subject were then entered into a two-way repeated-measures ANOVA, with factors of phrase-type ("Good news" and "I'm sorry to say"), and lag ( $p=1$ to 5). We also performed a follow-up three-way repeated measures ANOVA, in which means were concatenated over standardand HMM-MVAR analyses, and the analysis-type included as a further repeated-measures factor. Effect sizes are reported in units of partial and generalised eta-squared (Olejnik \& 
Algina, 2003; Bakeman, 2005). Post-hoc tests for the main effect of lag comprised a series of sequential t-tests comparing each subsequent increasing lag to the preceding one, subject to a Holm-Bonferroni correction for multiple comparisons. Effect sizes are reported in units of Hedges' $g_{a v}$ (Hedges \& Olkin, 1985; Lakens, 2013), whereby the pairwise mean difference is standardised by the average of each pairs' standard deviation and then corrected for bias.

All tests employed an alpha criterion of 0.05 for determining statistical significance.

\section{Results}

\section{Standard MVAR analyses}

We used a PCA-based active appearance model to capture key dimensions of facial variation in dynamic and natural expressions. We then used multivariate autoregressive (MVAR) models to predict PCA scores of future frames from the scores of the frames preceding them. Cross-validated prediction accuracy was assessed by correlating predicted and actual scores derived from the trailing frames.

We first employed a single-lag approach, in which a single pair of leading (predictor) and trailing (outcome) sequences were selected from each clip, separated by lags ranging from one frame $(40 \mathrm{~ms})$ up to five frames $(200 \mathrm{~ms})$. Figure $2 a$ shows the resulting distributions of cross-validated correlations between predicted and actual trailing PCA scores. Correlations appear high for shorter lags, but decrease as lags increase. We compared prediction accuracies against chance using a permutation testing procedure that randomly shuffled the temporal order of frames within the leading sequences. The empirical null distributions are illustrated in Figure S9, and corresponding statistics listed in Table S2. The true mean correlation exceeded the chance level (one-tailed test) for all phrase-types and lags in Subjects 1 and 3 (all $p<.05)$, and for both phrase-types but only the shortest two lags $(40 \mathrm{~ms}$, $80 \mathrm{~ms}$ ) in Subject 2 (all $p<.05$ ). We next compared correlations between the conditions via a two-way repeated measures ANOVA with factors of lag and phrase-type. This revealed a significant main effect of lag $\left(F(4,8)=136.04, p<.001, \eta_{P}^{2}=.99, \eta_{G}^{2}=.83\right)$, but no significant main effect of phrase-type $\left(F(1,2)=8.45, p=.101, \eta_{P}^{2}=.81, \eta_{G}^{2}=.09\right)$ nor phrase-type by lag interaction $\left(F(4,8)=0.84, p=.537, \eta_{P}^{2}=.30, \eta_{G}^{2}<.01\right)$. A series of sequential t-tests revealed the effect of lag was mediated by decreasing correlations with each increasing lag ( $40>80 \mathrm{~ms}$ : $\mathrm{t}(5)=24.41, p<.001$, Hedges' $g_{a v}=1.72 ; 80>120$ ms: $\mathrm{t}(5)=17.13, p<.001$, Hedges' $g_{a v}=$ 
$1.23 ; 120>160$ ms: $\mathrm{t}(5)=13.49, p<.001$, Hedges' $g_{a v}=0.92 ; 160>200$ ms: $\mathrm{t}(5)=11.76, p<$ .001 , Hedges' $g_{a v}=0.72$ ).
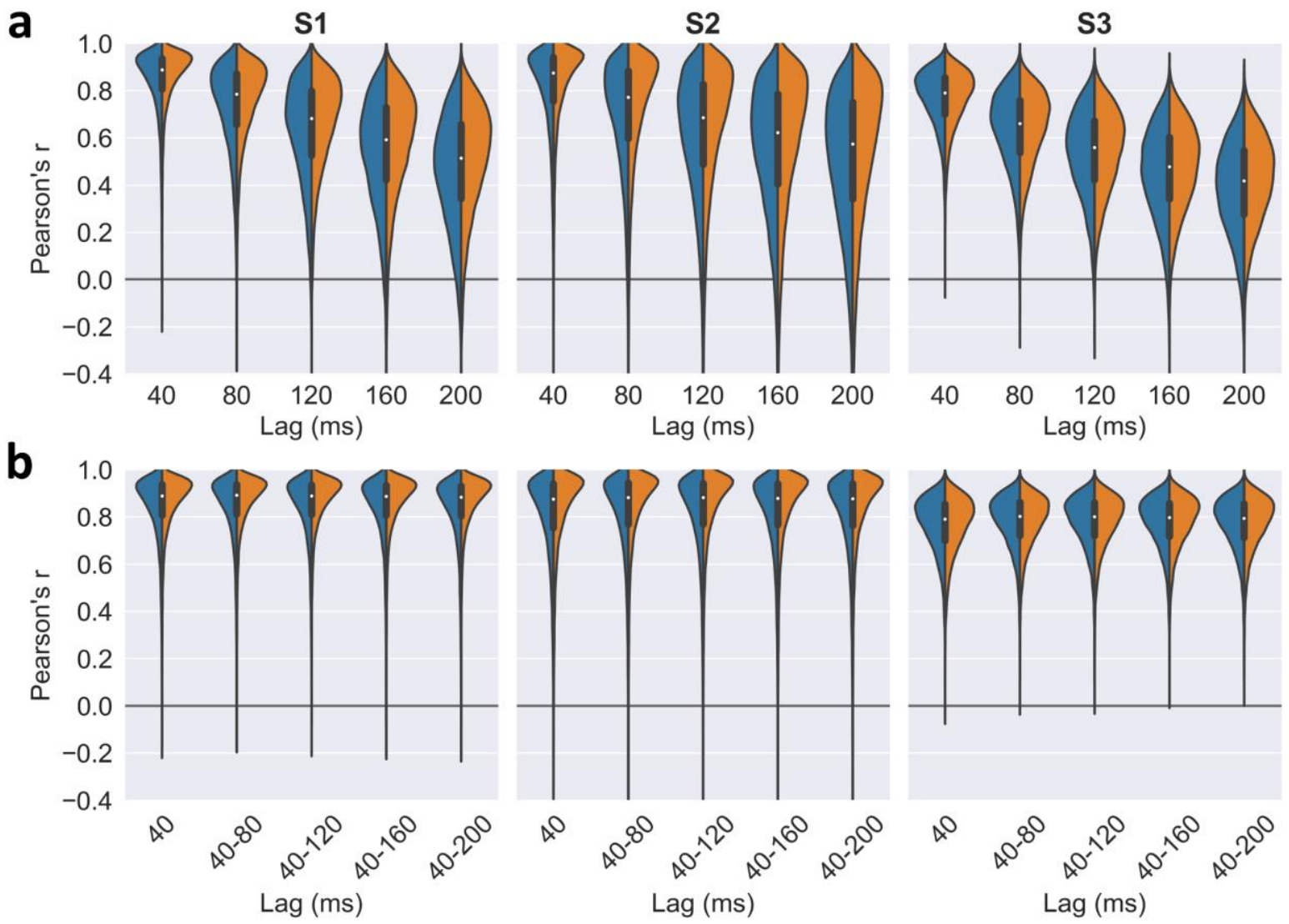

GN

ISTS

Figure 2. Standard-MVAR prediction accuracies for (a) single-lag and (b) multi-lag models. Violins show distributions of cross-validated correlations between predicted and actual trailing PCA scores for each subject, phrase-type (GN = "Good news", ISTS = "I'm sorry to say"), and lag. Inner boxplots show means and interquartile ranges collapsed over phrase-types.

We next employed a multi-lag approach, in which multiple leading sequences were selected from each clip, each offset by one frame. The predictor components therefore comprise multiple lags, the number of which determine the order of the model. If MVAR models are able to capture information about changes in the face over time, we would expect prediction accuracy to increase for higher-order models as the temporal support is extended. The resulting model correlations are shown in Figure $2 b$. Correlations remain high in all cases, but do not increase appreciably for higher order models. Permutation tests (Figure S10, Table S3) revealed that correlations were significantly greater than chance for all subjects, phrasetypes, and model-orders (all $p<.01$ ). Comparing between conditions, a repeated-measures 
ANOVA revealed no significant main effect of phrase-type $\left(F(1,2)=3.91, p=.187, \eta_{P}^{2}=.66\right.$, $\left.\eta_{G}^{2}=.06\right)$, but a significant main effect of $\operatorname{lag}\left(\mathrm{F}(4,8)=5.36, p=.021, \eta_{P}^{2}=.73, \eta_{G}^{2}=<.01\right)$ and phrase-type by lag interaction $\left(F(4,8)=12.18, p=.002, \eta_{P}^{2}=.86, \eta_{G}^{2}<.01\right)$. Post-hoc t-tests on the effect of lag revealed a slight increase in correlations from the first- to second-order models, $\left(40-80>40 \mathrm{~ms}: \mathrm{t}(5)=4.87, p=.009\right.$, Hedges' $\left.g_{a v}=0.13\right)$, but then decreasing correlations with further increasing model-orders $(40-80>40-120 \mathrm{~ms}: \mathrm{t}(5)=2.87, p=.035$, Hedges' $g_{a v}=0.03 ; 40-120>40-160$ ms: $\mathrm{t}(5)=6.18, p=.005$, Hedges' $g_{a v}=0.06 ; 40-160>40-$ $200 \mathrm{~ms}: \mathrm{t}(5)=7.50, p=.003$, Hedges' $\left.g_{a v}=0.06\right)$. Thus, including additional information about the temporal history of facial behaviours failed to substantially improve predictions of future behaviours, and indeed often had a deleterious effect instead.

To interrogate these results further, we plotted the regression coefficient matrices. Example matrices for the first cross-validation fold (train on even clips) and "Good news" phrase-type are shown in Figure 3, for all single-lag models and for the fifth-order multi-lag models. Figure S11 shows equivalent plots for the second- to fourth-order multi-lag models. Coefficients for odd clips of "Good news" phrases, and even and odd clips of "I'm sorry to say" phrases, all appeared similar (not shown). For single-lag models, coefficient matrices are dominated by the diagonal and appear close to the identity matrix. This implies a transformation that primarily duplicates the PCA scores of the leading frames into the trailing space. Similarly, the multi-lag models indicate a strong diagonal over the leading (predictor) components for the shortest lag (40ms), then lesser modulations for components relating to more distal lags. Again, this implies a solution that primarily duplicates the PCA scores of the most recent leading frame. This suggests the MVAR models' predictions are largely based on image similarity between consecutive frames, rather than capturing more nuanced temporal trajectories evolving over longer time periods. This is consistent with the observation that higher order multi-lag models failed to outperform lower order ones. 

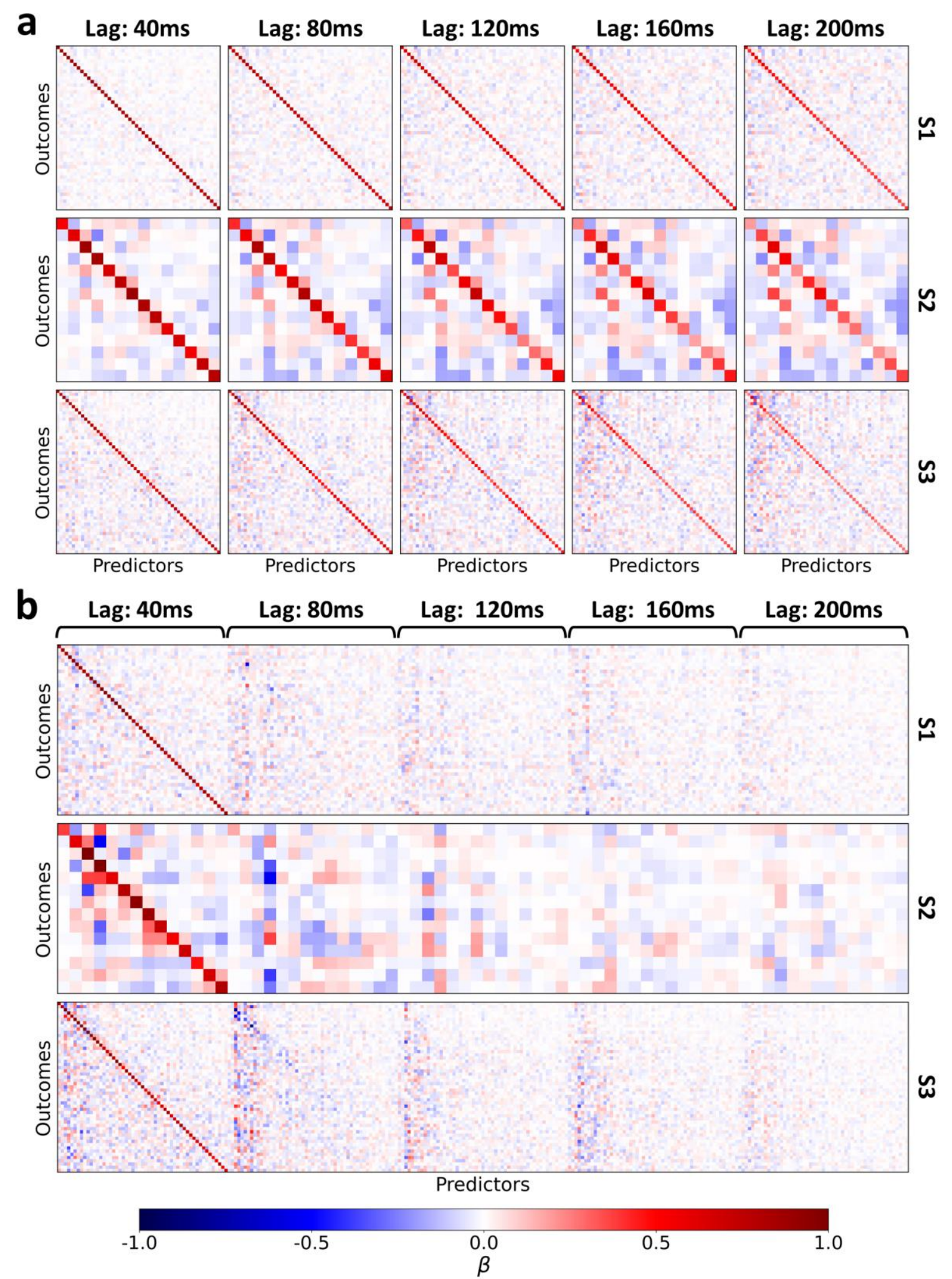

Figure 3. Standard-MVAR: example regression coefficient matrices (even clips of "Good news" phrases) for each subject. (a) Single-lag models. (b) Fifth-order multi-lag models: annotations indicate sections of matrix corresponding to each lag. Note that matrices have been transposed for display, such that trailing (outcome) components are displayed on rows and leading (predictor) components on columns. 


\section{Identifying Behavioural States}

Each video sequence comprised a wide variety of facial behaviours. Potentially, the failure of the MVAR models to extract more nuanced temporal information may be attributed to challenges in capturing dynamics of all these behaviours within a single model. To this end, we used Hidden Markov Models (HMMs) to identify underlying behavioural states in the facial expressions over time, in order to generate sequences containing more consistent facial behaviours for further MVAR analyses.

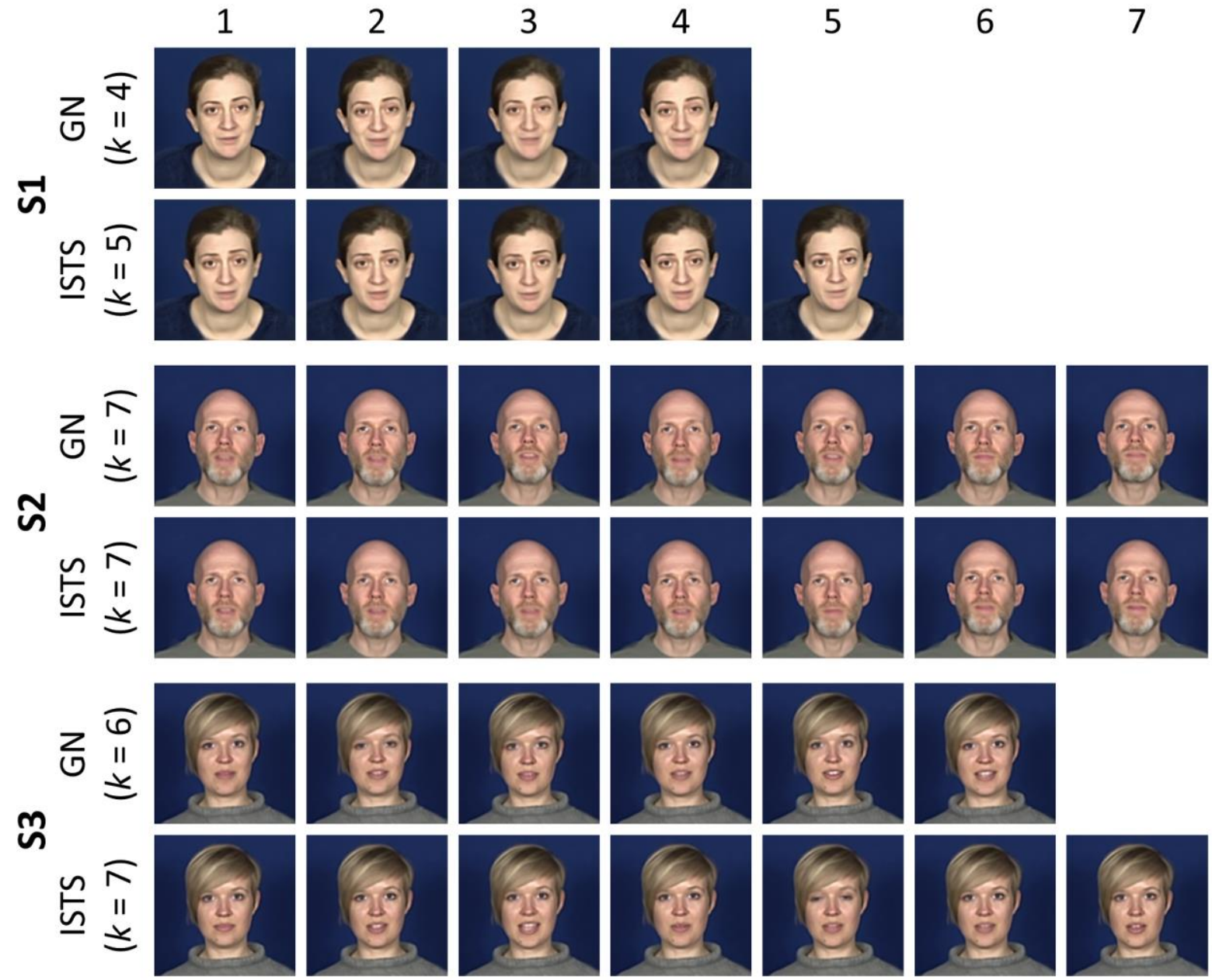

Figure 4. Back-projections of full-dataset HMM state means to the image space, for each subject and phrase-type (GN = "Good news", ISTS = "I'm sorry to say").

We first fit HMMs to the full dataset of PCA scores (without cross-validation). The number of states was chosen independently for each subject and phrase-type (see Methods). To visualise the facial behaviours embodied by each state, we back-projected the state-mean vectors to the image space. Visualisations for the selected models are shown in Figure 4, and 
visualisations for all models are shown in Figures S2-S7. Each state captures distinct facial behaviours, including changes in head pose and expressions such as smiles, grimaces, frowns, blinks, speech-related lip movements, and neutral/resting expressions. Differences in the emotional valence of the expressions are also evident between the two phrase-types. States also appear more distinct for Subjects 2 and 3 than for Subject 1 . Anecdotally, Subject 1 displayed a greater range of expression which may have been more challenging for the HMMs to capture.

We next examined the state probabilities. Figure $5 a$ shows the state transition matrices, illustrating the probability of each state given the state of the preceding frame. Each matrix has a strong diagonal demonstrating that consecutive frames mostly share the same state, although weaker off-diagonal elements indicate occasional transitions between states. This implies relatively slow changes between behavioural states over time - relative to the $25 \mathrm{fps}$ (40 ms) sampling rate. To further interrogate the time course of each state, we extracted state posterior probabilities over frames and binned the values by normalised clip duration. The resulting time courses are illustrated in Figure $5 b$. Most states show clear modulations of posterior probabilities over time. For instance, Subject 3's "Good news" probabilities reveal state 3 is primarily associated with early time points. The corresponding back-projected state mean (Figure 4) illustrates a neutral expression, consistent with a resting facial state prior to beginning the vocalisation. Meanwhile states 1, 5, and 6 are more common in later time windows, each of which illustrate various gradations of smiles and speech-related lip movements, consistent with the ongoing vocalisation. To visualise these changes more clearly, we created animations by weighting the back-projected state means according to the posterior probability time courses (Videos S1-S6). All of these animations capture an initial movement of the head as the vocalisation begins. Similarly, some reconstructions also display an opening of the mouth around the onset of vocalisations. Further changes in head posture and expression are also evident over time, particularly for Subject's 2 and 3. Taken together, these results suggest the HMMs successfully captured underlying basis states of the facial behaviours over time. 
a
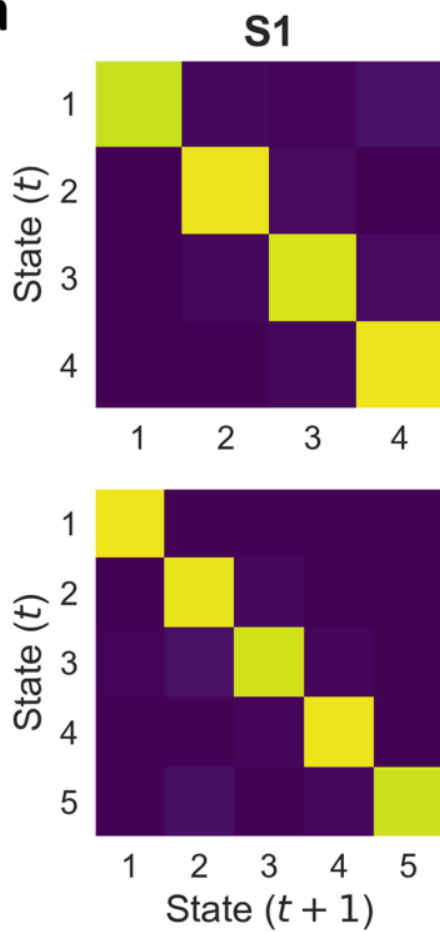

b

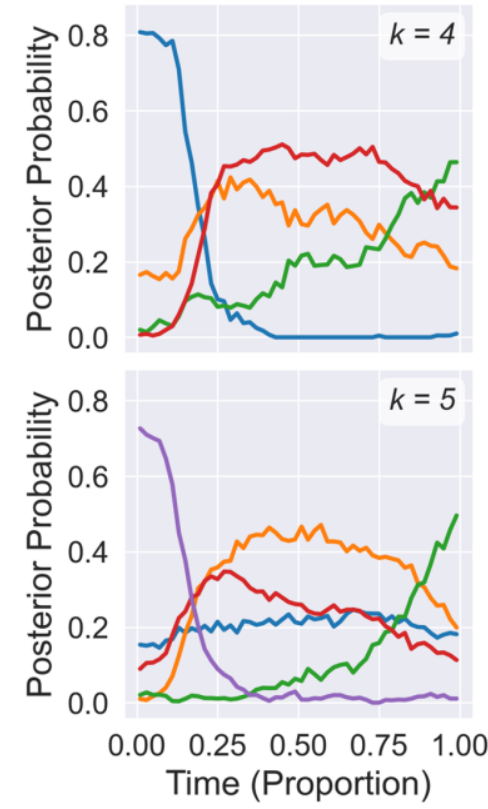

S2
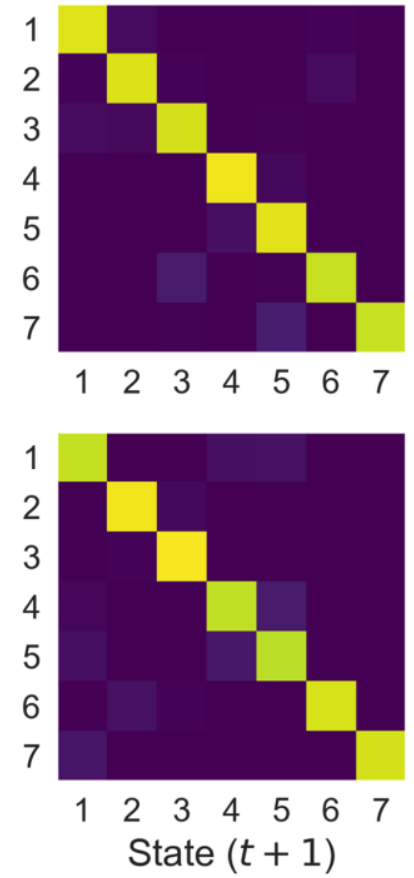

S2

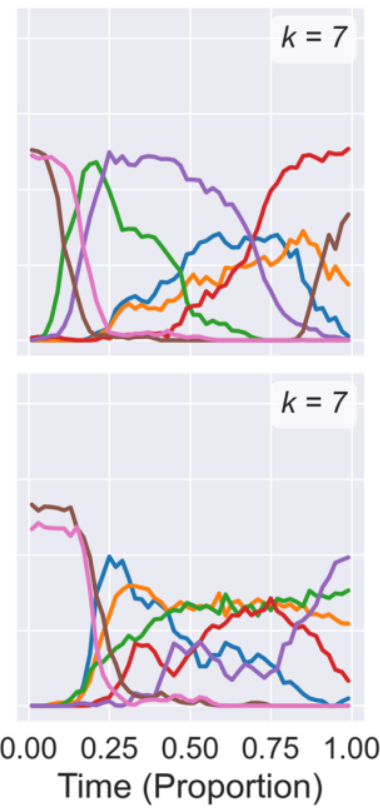

S3

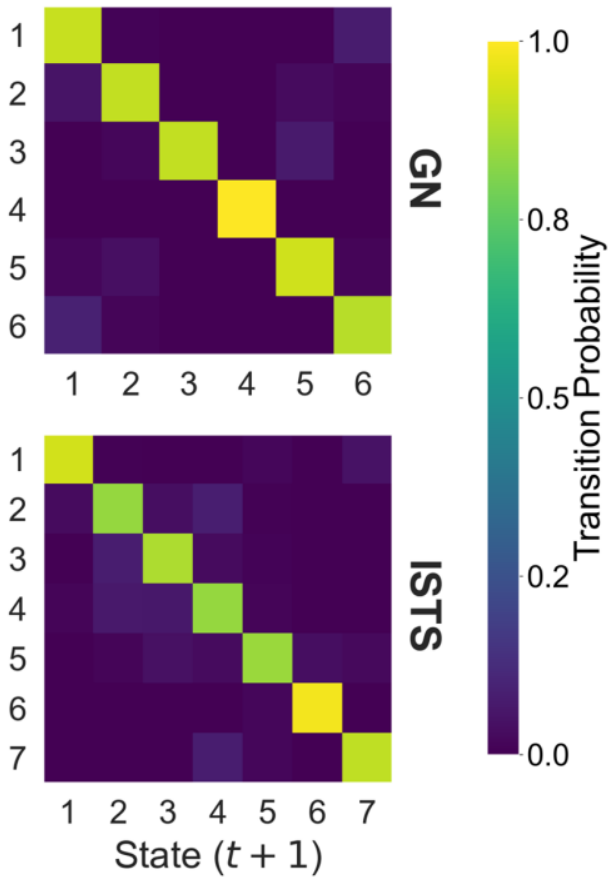

S3

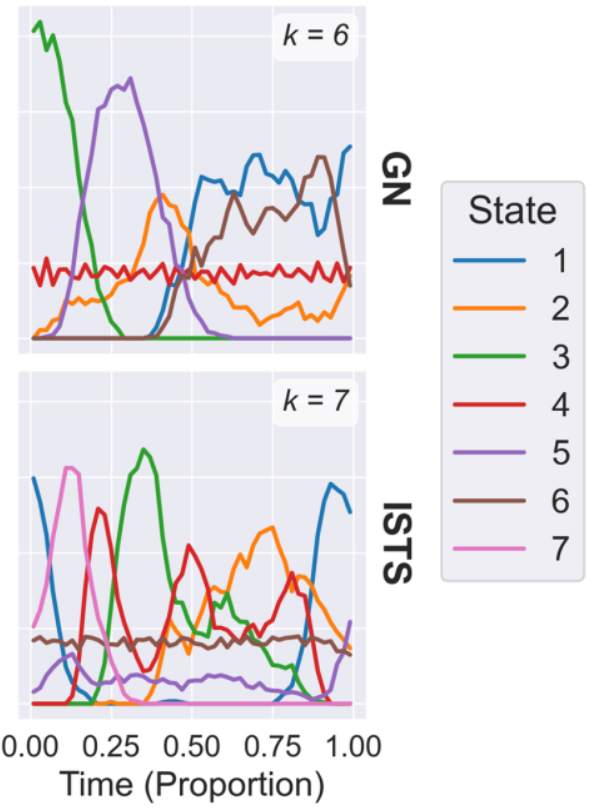

Figure 5. Full-dataset HMM state probabilities for each subject and phrase-type (GN = "Good news", ISTS = "I'm sorry to say"). (a) State transition probabilities. (b) Time courses of posterior probabilities for each state plotted over normalised clip durations.

Finally, we applied the HMMs to the cross-validated PCA scores (even and odd clips separately). Back-projected state means are illustrated in Figure S12. HMMs again extracted a number of distinct behavioural states, however not all states fully reproduced across the data splits. For instance, Subject 3's "Good news" states reveal a blinking expression for odd 
clips (state 2) that is not present for even clips, while only a partial blink is evident in the full dataset (state 2). However other expressions do more clearly reproduce, such as the "broad smile" expression in the same examples (full dataset: state 6, even clips: state 4, odd clips: state 3). Thus, while the HMMs are successful at extracting distinct behavioural states, the exact states identified are somewhat dependent on the specific content of the training data.

\section{HMM-MVAR analyses}

Finally, we combined the MVAR and HMM approaches. We identified sequences of frames from each HMM state, which were then further split into leading and trailing frame sequences and submitted to MVAR analyses for each state independently. This was performed within the cross-validation scheme, such that both the HMM and MVAR models were trained on data from one of the splits (odd or even clips) and tested on data from the other split.

We first tested the single-lag approach. Cross-validated correlations are shown in Figure $6 a$. Permutation tests (Figure S13; Table S4) revealed the mean correlations did not exceed the chance level for any subject, phrase-type, or lag (all $p>.05$ ). This apparent reduction in performance compared to the standard-MVAR approach was driven both by a reduction in true correlations and an increase in correlations under the empirical null distribution (cf. Figure S9 and Figure S13). Because sequences within a given state contain more consistent facial behaviours, differences between frames over time are reduced, and so randomising the order of frames does not have so much of a disruptive effect. We then compared correlations between conditions. A repeated-measures ANOVA revealed a significant main effect of phrase-type $\left(F(1,2)=48.76, p=.020, \eta_{P}^{2}=.96, \eta_{G}^{2}=.07\right)$ due to overall higher correlations for "I'm sorry to say" than "Good news" phrases. There was also a significant main effect of lag $\left(F(4,8)=103.65, p<.001, \eta_{P}^{2}=.98, \eta_{G}^{2}=.84\right)$, but the phrase-type by lag interaction was not significant $\left(F(4,8)=0.10, p=.979, \eta_{P}^{2}=.05, \eta_{G}^{2}<.01\right)$. Post-hoc t-tests revealed the effect of lag was driven by decreasing correlations with increasing lags (40>80 ms: $\mathrm{t}(5)=20.05, p<$ .001 , Hedges' $g_{a v}=1.72 ; 80>120 \mathrm{~ms}: \mathrm{t}(5)=16.50, p<.001$, Hedges' $g_{a v}=1.30 ; 120>160 \mathrm{~ms}$ : $\mathrm{t}(5)=12.15, p<.001$, Hedges' $g_{a v}=0.99 ; 160>200$ ms: $\mathrm{t}(5)=9.41, p<.001$, Hedges' $g_{a v}=$ 0.74). Thus, results appeared similar to the standard-MVAR analyses in that correlations decreased with increasing lags, although correlations no longer exceeded the empirical chance level. 
a
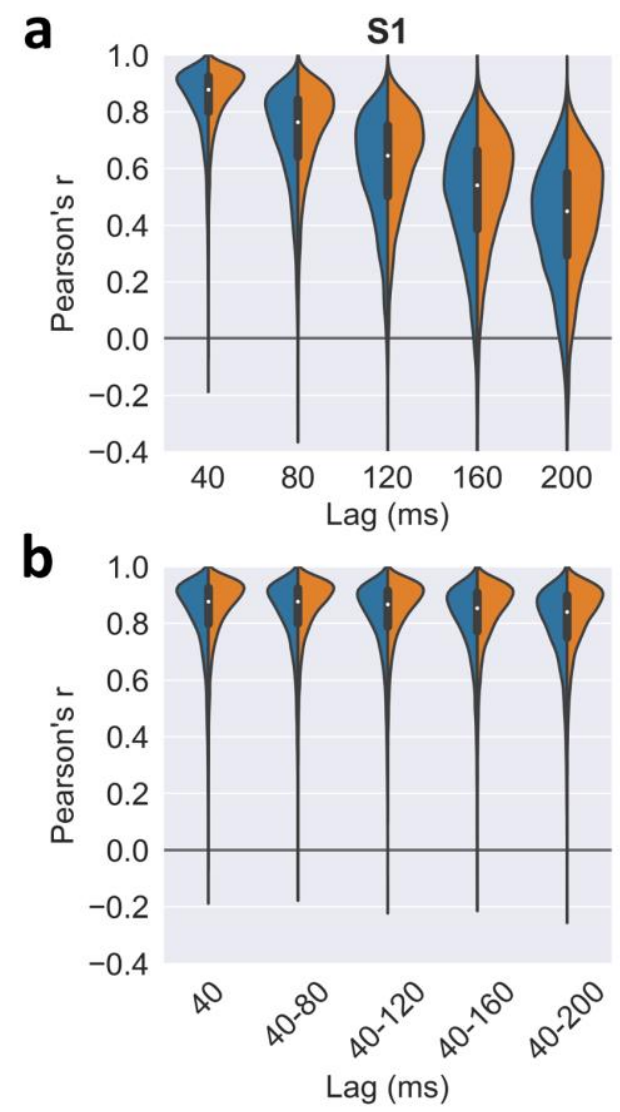
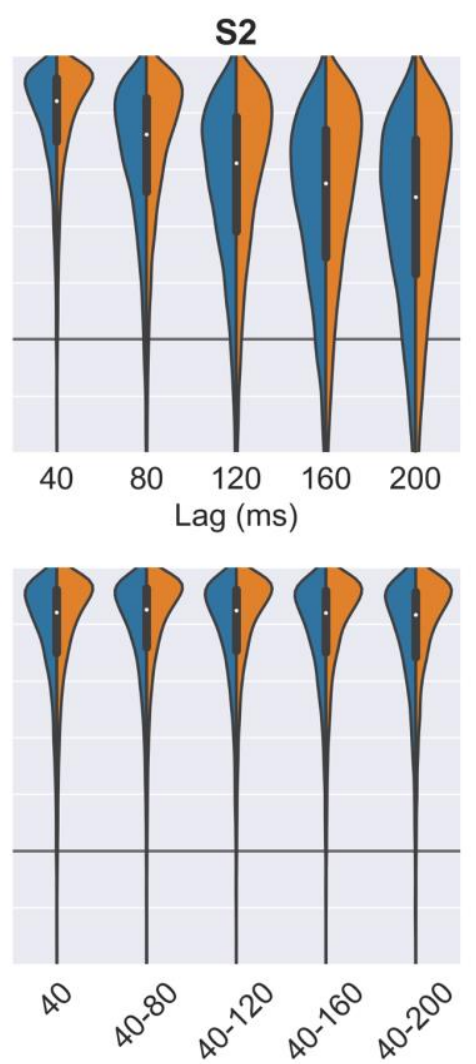

Lag (ms)

GN

\section{ISTS}
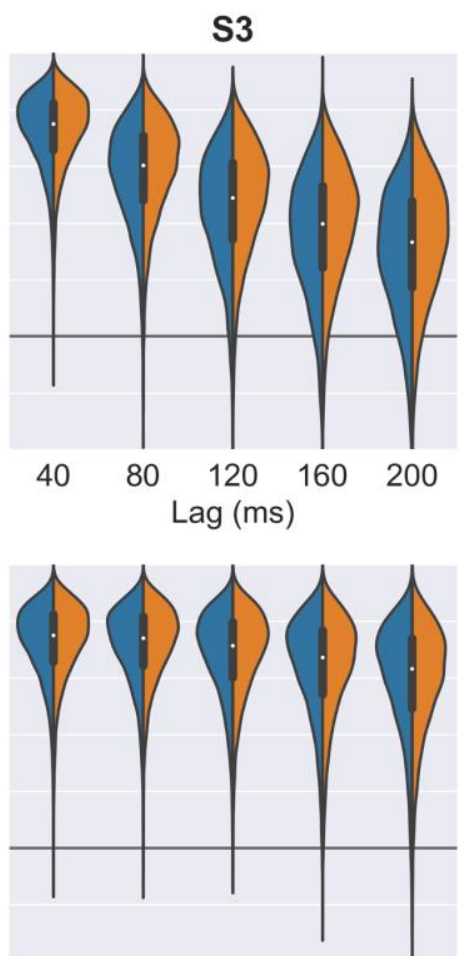

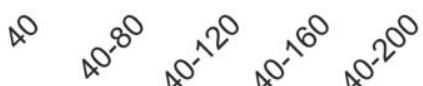

$\operatorname{Lag}(\mathrm{ms})$

Figure 6. HMM-MVAR prediction accuracies for (a) single-lag and (b) multi-lag models. Violins show distributions of cross-validated correlations between predicted and actual trailing PCA scores for each subject, phrase-type (GN = "Good news", ISTS = "I'm sorry to say"), and lag. Inner boxplots show means and interquartile ranges collapsed over phrase-types.

We next tested the multi-lag approach. If the MVAR models are able to capture more nuanced temporal information now that sequences comprise more consistent facial behaviours, then correlations should now increase for higher order models. The cross-validated correlation distributions are illustrated in Figure 6b. Permutation tests (Figure S14, Table S5) revealed mean correlations were significantly greater than the empirical chance level for all phrasetypes and lags for Subject 1 (all $p<.05$ ), but no comparisons passed significance for Subjects 2 or 3 (all $p>.05$ ). Similar to the single-lag approach, this apparent reduction in performance relative to the standard-MVAR analyses was due to both a reduction in the true correlations and an increase in the correlations under the empirical null distribution. We next compared mean correlations between conditions. A repeated-measures ANOVA revealed a significant main effect of lag $\left(F(4,8)=7.28, p=.009, \eta_{P}^{2}=.78, \eta_{G}^{2}=.08\right)$, but no significant main effect of 
phrase-type $\left(\mathrm{F}(1,2)=4.40, p=.171, \eta_{P}^{2}=.69, \eta_{G}^{2}=.03\right)$ nor phrase-type by lag interaction $\left(F(4,8)=0.29, p=.878, \eta_{P}^{2}=.13, \eta_{G}^{2}<.01\right)$. Post-hoc t-tests on the main effect of lag revealed no significant difference between the first- and second-order models $(t(5)=0.23, p=.829$, Hedges' $\left.g_{a v}=0.01\right)$, but correlations then decreased across higher order models $(40-80>40-$ 120 ms: $\mathrm{t}(5)=4.82, p=.013$, Hedges' $g_{a v}=0.17 ; 40-120>40-160 \mathrm{~ms}: \mathrm{t}(5)=4.93, p=.013$, Hedges' $g_{a v}=0.21 ; 40-160>40-200 \mathrm{~ms}: \mathrm{t}(5)=6.33, p=.006$, Hedges' $\left.g_{a v}=0.17\right)$.

Finally, to compare between the standard- and HMM-MVAR analyses, we concatenated mean correlations over the analyses and repeated our ANOVAs with the addition of the analysis-type as a repeated-measures factor. For the single-lag approach, this revealed a significant main effect of analysis-type $\left(F(1,2)=62.60, p=.016, \eta_{P}^{2}=.97, \eta_{G}^{2}=.17\right)$ due to overall higher correlations for the standard- than HMM-MVAR analyses. There was also a significant main effect of lag $\left(F(4,8)=118.68, p<.001, \eta_{P}^{2}=.98, \eta_{G}^{2}=.84\right)$ and analysis-type by phrase-type by lag interaction $\left(F(4,8)=3.99, p=.048, \eta_{P}^{2}=.66, \eta_{G}^{2}<.01\right)$, while the main effect of phrase-type approached significance $\left(\mathrm{F}(1,2)=15.99, p=.057, \eta_{P}^{2}=.89, \eta_{G}^{2}=.08\right)$. None of the remaining interactions were significant (all $p<.05$ ). Post-hoc t-tests revealed the main effect of lag was mediated by decreasing correlations with increasing lags (40>80 ms: $\mathrm{t}(11)=32.44, p<.001$, Hedges' $g_{a v}=1.87 ; 80>120 \mathrm{~ms}: \mathrm{t}(11)=24.81, p<.001$, Hedges $^{\prime} g_{a v}=$ $1.34 ; 120>160 \mathrm{~ms}: \mathrm{t}(11)=18.91, p<.001$, Hedges' $g_{a v}=0.98 ; 160>200$ ms: $\mathrm{t}(11)=15.39, p$ $<.001$, Hedges' $\left.g_{a v}=0.74\right)$.

Similarly, for the multi-lag approach, there was again a significant main effect of analysis-type $\left(F(1,2)=26.33, p=.036, \eta_{P}^{2}=.93, \eta_{G}^{2}=.19\right)$ due to overall higher correlations for the standardthan HMM-MVAR analyses. There was also a significant main-effect of lag $(F(4,8)=9.14, p=$ $\left..004, \eta_{P}^{2}=.82, \eta_{G}^{2}=.03\right)$, and a significant analysis-type by lag interaction $(F(4,8)=5.37, p=$ $\left..021, \eta_{P}^{2}=.73, \eta_{G}^{2}=.02\right)$. The main effect of phrase-type was not significant $(F(1,2)=4.20, p$ $=.177, \eta_{P}^{2}=.68, \eta_{G}^{2}=.04$ ) and nor were the remaining interactions (all $p<.05$ ). Post-hoc t-tests on the main effect of lag revealed a non-significant trend for higher correlations in second- than first-order models (40-80 > $40 \mathrm{~ms}: \mathrm{t}(11)=2.15, p=.055$, Hedges' $\left.g_{a v}=0.07\right)$, but correlations then decreased across higher-order models $(40-80>40-120 \mathrm{~ms}: \mathrm{t}(11)=3.59, p=$ .008 , Hedges' $g_{a v}=0.12 ; 40-120>40-160 \mathrm{~ms}: \mathrm{t}(11)=3.90, p=.007$, Hedges' $g_{a v}=0.15 ; 40-$ $160>40-200$ ms: $\mathrm{t}(11)=4.52, p=.003$, Hedges' $\left.g_{a v}=0.12\right)$. 
Thus, the HMM-MVAR analyses again failed to predict the time courses of the facial behaviours beyond image similarity, and indeed actually performed slightly poorer than the equivalent standard-MVAR analyses.

\section{Discussion}

In this study we tested whether temporal information about the recent history of natural and dynamic facial behaviours can be used to predict future behaviours. We used a PCA-based active appearance model to capture critical dimensions of facial variation over time. We then employed multivariate autoregressive (MVAR) models to predict PCA scores of future frames from the frames immediately preceding them. While we were able to predict future frames, these predictions appeared to be primarily based on image similarity between consecutive frames. We also used Hidden Markov Models (HMMs) to identify underlying basis states of the dynamic facial behaviours. These models successfully extracted distinct behavioural states from the frame sequences, but MVAR models operating within each state did not perform better than their counterparts operating on the full clips. We therefore found no evidence that autoregressive models applied to a PCA face space can predict the time course of dynamic facial behaviours beyond one or two frames.

We elicited natural and dynamic facial expressions from people vocalising short sentences conveying good or bad news. We employed a multiple-channel gradient model (Johnston et al., 1999; Cowe, 2003) to measure changes in form and texture over frames, and then used principal components analysis to capture key dimensions of variation. In a previous study, we demonstrated that this model supported both the discrimination and reconstruction of the emotional valance of the expressions (Watson et al., 2020). However, this analysis did not consider the temporal relationships within these representations. Here, we applied cross-validated MVAR models (Harrison et al., 2003; Hytti et al., 2006) to these representations with the aim of using the immediate history of facial behaviours to predict future behaviours. We employed two MVAR approaches: single-lag models predicted trailing frames using a single set of leading frames separated by a given lag, while multi-lag models measured the predictive ability obtained by including all leading sequences up to the trailing frames. The single-lag approach revealed reduced prediction accuracies as the lag between 
leading and trailing frames increased, indicating increasing dissimilarity between representations at more distal timepoints. The multi-lag approach revealed high prediction accuracies, but these did not substantially improve for higher-order models that extended the temporal support. Small improvements were sometimes seen from first to second-order models, however further increasing the model-order had a deleterious effect. This indicates that information represented at more distal timepoints provided very little predictive benefit over that already given by more recent frames. Rather, including additional temporal information actually impaired model generalisation between data splits. Inspection of the regression coefficient matrices indicated strong diagonals over predictor components for the most recent lag, implying a solution largely reliant on duplicating PCA scores from the preceding frame. For MVAR models applied to the full clips, performance often exceeded the chance level determined by permuting the temporal order of frames. This might suggest some sensitivity to temporal order, but it is important to note that this permutation scheme also disrupts image similarity between leading and trailing sequences as consecutive frames are no longer guaranteed to be consecutive. Indeed, correlations under the empirical null distributions were increased for the HMM generated sequences where frames were more similar within each state, and so permutating their order had a less disruptive effect on image similarity. In fact, performance often failed to exceed chance levels for these sequences.

One possible explanation for the poor performance of the MVAR models is that the dynamic sequences comprise a diverse array of facial behaviours, and it may be overly challenging for a single autoregressive model to capture all of these simultaneously. To investigate this possibility, we used HMMs to automatically segment the clips into distinct behavioural states. By extracting frames from each state, we constructed sequences that comprised more selective ranges of facial behaviours. Nevertheless, we found that MVAR models trained on sequences within each state failed to outperform those trained on the full clips, and indeed actually showed significantly worse prediction accuracy. Thus, the relative consistency or inconsistency of facial behaviours within the frame sequences cannot explain the failure of the MVAR approach. Nevertheless, the HMMs did prove successful in extracting underlying behavioural states. Back-projections of the state means revealed perceptually distinct and interpretable facial behaviours, including different head poses, expressions, and speech movements. Our results did highlight some potential challenges with this approach. Firstly, 
the states appeared only partially reproducible across cross-validation folds, indicating the precise content of the training data is important to consider. Secondly, the extracted states appeared more distinctive for Subjects 2 and 3 than for Subject 1, indicating potentially variable model performances between subjects, and again suggesting outputs are closely linked to the input data. Finally, these methods require choosing a number of states to extract, which is an inherently subjective choice even if objective measures are used to assist in this. Nevertheless, different models fit to the same data did tend to extract similar behavioural states even as the number of extracted states varied (Figures S2-S7). Thus, the HMM approach described here may prove useful for automated extraction of facial behaviours.

The failure to see a benefit from incorporating additional frames contrasts with a recent publication from our group (Scholes et al., 2020). In that study, a PCA-based approach similar to the one described here was used to predict MR vocal tract images from corresponding video recorded facial movements, and vice versa. Scholes et al. showed that including additional temporal information improved prediction accuracy, indicating that incorporating information about the direction of change can, for example, disambiguate whether the jaw is rising or dropping which could then help to link the video and MR information. However, the approach employed by Scholes et al. differs from the current study in that predictions were of spatial configurations between two different modalities (video and MRI), rather than explicitly predicting changes over time.

The current results may seem surprising because there is substantial evidence for perceptual advantages for dynamic over static faces amongst human observers (O'Toole et al., 2002; O'Toole \& Roark, 2010). Behaviourally, people are better at discriminating both facial identities and expressions from dynamic than static faces (Lander \& Butcher, 2015). Similarly, multiple brain regions in the face processing network display preferential responses for dynamic over static face stimuli (Bernstein \& Yovel, 2015). Thus, temporal information is clearly present within dynamic faces, and can be extracted by the human perceptual system. Why then do autoregressive models not similarly capture such information? One possibility is that while temporal information may be present, it may not be predictive. For instance, while a given temporal sequence of facial behaviours as a whole may provide discriminative information not present in static images, transient points within that sequence may not be 
sufficiently related to enable prediction from one timepoint to another. Another possibility is that temporal information is both present and predictive, but the computational approaches employed here are insensitive to it. For instance, MVAR models may be unable to properly capture temporal trajectories across the PCA face space. MVAR models have found widespread application in many other fields (Sims, 1980; Richardson, 1981; Harrison et al., 2003), and so do represent a sensible choice for temporal forecasting, but may nevertheless be insufficient for the current task.

In conclusion, we tested whether multivariate autoregressive models applied to a PCA-based face model could be used to extract and predict the time course of dynamic and natural facial behaviours. While we were able to predict future frames from those preceding them, these predictions did not extend beyond image similarity between consecutive frames, and hence did not capture more nuanced time courses evolving over longer periods. Hidden Markov models proved successful at extracting distinct states underlying facial behaviours, though this did not improve autoregression performance. Thus, we found no evidence for autoregressive models having predictive power in the context of dynamic facial behaviours.

\section{Conflicts of Interest}

The authors declare no conflicts of interest.

\section{Author Contributions}

Both authors conceived and developed the experiments. D.M.W. performed the data analysis under the supervision of A.J. Both authors contributed to the writing of the manuscript and approved the final version of the manuscript for submission.

\section{Funding}

This research was funded by the NIHR Nottingham Biomedical Research Centre and supported by the NIHR Nottingham Clinical Research Facilities. The views represented are the views of 
the authors alone and do not necessarily represent the views of the Department of Health in England, NHS, or the National Institute for Health Research.

\section{Acknowledgements}

We would like to thank Ben B. Brown for performing the original data collection.

\section{Data Availability}

Code, datasets, and supplementary material for this study can be found on the Open Science Framework (https://osf.io/wbm57/).

\section{References}

Bakeman, R. (2005). Recommended effect size statistics for repeated measures designs. Behavior Research Methods, 37(3), 379-384.

Bernstein, M., \& Yovel, G. (2015). Two neural pathways of face processing: A critical evaluation of current models. Neuroscience \& Biobehavioral Reviews, 55, 536-546.

Bould, E., \& Morris, N. (2008). Role of motion signals in recognizing subtle facial expressions of emotion. British Journal of Psychology, 99(2), 167-189.

Bruce, V., \& Young, A. (1986). Understanding face recognition. British Journal of Psychology, 77(3), 305-327.

Calder, A. J., Burton, A. M., Miller, P., Young, A. W., \& Akamatsu, S. (2001). A principal component analysis of facial expressions. Vision Research, 41(9), 1179-1208.

Cao, Q., Shen, L., Xie, W., Parkhi, O. M., \& Zisserman, A. (2018). VGGFace2: A Dataset for Recognising Faces across Pose and Age. In 2018 13th IEEE International Conference on Automatic Face \& Gesture Recognition (FG 2018) (pp. 67-74). IEEE.

Celeux, G., \& Durand, J.-B. (2008). Selecting hidden Markov model state number with crossvalidated likelihood. Computational Statistics, 23(4), 541-564.

Cowe, G. (2003). Example-based computer-generated facial mimicry. University College London.

Ellis, H. D. (1975). Recognizing Faces. British Journal of Psychology, 66(4), 409-426.

Furl, N., Henson, R. N., Friston, K. J., \& Calder, A. J. (2015). Network Interactions Explain Sensitivity to Dynamic Faces in the Superior Temporal Sulcus. Cerebral Cortex, 25(9), 2876-2882. 
Harrison, L., Penny, W. D., \& Friston, K. (2003). Multivariate autoregressive modeling of fMRI time series. Neurolmage, 19(4), 1477-1491.

Hedges, L. V., \& Olkin, I. (1985). Statistical Methods for Meta-Analysis. San Diego, CA: Academic Press.

Holm, S. (1979). A Simple Sequentially Rejective Multiple Test Procedure. Scandinavian Journal of Statistics, 6(2), 65-70.

Hytti, H., Takalo, R., \& Ihalainen, H. (2006). Tutorial on Multivariate Autoregressive Modelling. Journal of Clinical Monitoring and Computing, 20(2), 101-108.

Jenkins, R., White, D., Van Montfort, X., \& Mike Burton, A. (2011). Variability in photos of the same face. Cognition, 121(3), 313-323.

Johnston, A., McOwan, P. W., \& Benton, C. P. (1999). Robust velocity computation from a biologically motivated model of motion perception. Proceedings of the Royal Society of London. Series B: Biological Sciences, 266(1418), 509-518.

Johnston, A., McOwan, P. W., \& Buxton, H. (1992). A computational model of the analysis of some first-order and second-order motion patterns by simple and complex cells. Proceedings of the Royal Society B: Biological Sciences, 250(1329), 297-306.

Knight, B., \& Johnston, A. (1997). The Role of Movement in Face Recognition. Visual Cognition, 4(3), 265-273.

Lakens, D. (2013). Calculating and reporting effect sizes to facilitate cumulative science: a practical primer for t-tests and ANOVAs. Frontiers in Psychology, 4(NOV), 1-12.

Lander, K., \& Butcher, N. (2015). Independence of face identity and expression processing: exploring the role of motion. Frontiers in Psychology, 6(MAR), 1-6.

Lander, K., Christie, F., \& Bruce, V. (1999). The role of movement in the recognition of famous faces. Memory \& Cognition, 27(6), 974-985.

Lander, K., \& Chuang, L. (2005). Why are moving faces easier to recognize? Visual Cognition, 12(3), 429-442.

O’Toole, A. J., Roark, D. A., \& Abdi, H. (2002). Recognizing moving faces: a psychological and neural synthesis. Trends in Cognitive Sciences, 6(6), 261-266.

O'Toole, A., \& Roark, D. (2010). Memory for Moving Faces: The Interplay of Two Recognition Systems. In Dynamic Faces (pp. 15-30). The MIT Press.

Olejnik, S., \& Algina, J. (2003). Generalized Eta and Omega Squared Statistics: Measures of Effect Size for Some Common Research Designs. Psychological Methods, 8(4), 434-447.

Oosterhof, N. N., \& Todorov, A. (2008). The functional basis of face evaluation. Proceedings of the National Academy of Sciences, 105(32), 11087-11092.

Pike, G. E., Kemp, R. I., Towell, N. A., \& Phillips, K. C. (1997). Recognizing Moving Faces: The Relative Contribution of Motion and Perspective View Information. Visual Cognition, 4(4), 409-438.

Pitcher, D., Dilks, D. D., Saxe, R. R., Triantafyllou, C., \& Kanwisher, N. (2011). Differential 
selectivity for dynamic versus static information in face-selective cortical regions. Neurolmage, 56(4), 2356-2363.

Pohle, J., Langrock, R., van Beest, F. M., \& Schmidt, N. M. (2017). Selecting the Number of States in Hidden Markov Models: Pragmatic Solutions Illustrated Using Animal Movement. Journal of Agricultural, Biological and Environmental Statistics, 22(3), 270293.

Richardson, C. W. (1981). Stochastic simulation of daily precipitation, temperature, and solar radiation. Water Resources Research, 17(1), 182-190.

Roether, C. L., Omlor, L., Christensen, A., \& Giese, M. A. (2009). Critical features for the perception of emotion from gait. Journal of Vision, 9(6), 1-32.

Rosenblum, L. D., Johnson, J. A., \& Saldaña, H. M. (1996). Point-Light Facial Displays Enhance Comprehension of Speech in Noise. Journal of Speech, Language, and Hearing Research, 39(6), 1159-1170.

Rosenblum, L. D., \& Saldaña, H. M. (1996). An audiovisual test of kinematic primitives for visual speech perception. Journal of Experimental Psychology: Human Perception and Performance, 22(2), 318-331.

Scholes, C., Skipper, J. I., \& Johnston, A. (2020). The interrelationship between the face and vocal tract configuration during audiovisual speech. Proceedings of the National Academy of Sciences, 117(51), 32791-32798.

Schultz, J., \& Pilz, K. S. (2009). Natural facial motion enhances cortical responses to faces. Experimental Brain Research, 194(3), 465-475.

Schwarz, G. (1978). Estimating the Dimension of a Model. The Annals of Statistics, 6(2), 461464.

Sims, C. A. (1980). Macroeconomics and Reality. Econometrica, 48(1), 1.

Sliwinska, M. W., Bearpark, C., Corkhill, J., McPhillips, A., \& Pitcher, D. (2020). Dissociable pathways for moving and static face perception begin in early visual cortex: Evidence from an acquired prosopagnosic. Cortex, 130, 327-339.

Trautmann, S. A., Fehr, T., \& Herrmann, M. (2009). Emotions in motion: Dynamic compared to static facial expressions of disgust and happiness reveal more widespread emotionspecific activations. Brain Research, 1284, 100-115.

Turk, M., \& Pentland, A. (1991). Eigenfaces for Recognition. Journal of Cognitive Neuroscience, 3(1), 71-86.

Watson, D. M., Brown, B. B., \& Johnston, A. (2020). A data-driven characterisation of natural facial expressions when giving good and bad news. PLOS Computational Biology, 16(10), e1008335.

Zhang, H., Japee, S., Stacy, A., Flessert, M., \& Ungerleider, L. G. (2020). Anterior superior temporal sulcus is specialized for non-rigid facial motion in both monkeys and humans. Neurolmage, 218(October 2019), 116878. 


\section{Supplementary Figure $\&$ Video Captions}

\section{Supplementary Figures}

Figure S1. HMM prediction errors across numbers of states $(k)$ for each subject and phrasetype (GN = "Good news", ISTS = "I'm sorry to say"). Double y-axes illustrate BIC values for fulldataset (without cross-validation) (green, left axes), and cross-validated negative loglikelihoods (for testing data portions) (red, right axes). Larger symbols indicate minimal prediction errors, and hence recommended values of $k$.

Figure S2. Subject 1, "Good news": all back-projected HMM state means for full-datasets for values of $k=2$ to 10 . To aid visualisation, images have been sorted to match state orders between consecutive values of $k$ by maximising pairwise correlations of state mean vectors. Annotations in top right indicate state indices.

* Selected model, † Optimal BIC (full-dataset), ‡ Optimal cross-validated likelihood

Figure S3. Subject 1, "I'm sorry to say": all back-projected HMM state means for full-datasets for values of $k=2$ to 10 . Other details as per Figure S2.

Figure S4. Subject 2, "Good news": all back-projected HMM state means for full-datasets for values of $k=2$ to 10 . Other details as per Figure $S 2$.

Figure S5. Subject 2, "I'm sorry to say": all back-projected HMM state means for full-datasets for values of $k=2$ to 10 . Other details as per Figure S2.

Figure S6. Subject 3, "Good news": all back-projected HMM state means for full-datasets for values of $k=2$ to 10 . Other details as per Figure S2.

Figure S7. Subject 3, "I'm sorry to say": all back-projected HMM state means for full-datasets for values of $k=2$ to 10 . Other details as per Figure S2.

Figure 58. Percentage of frames (across clips) allocated to cross-validated HMM state sequences, for each subject and phrase-type (GN = "Good news", ISTS = "I'm sorry to say"). Each frame is allocated to the state with the greatest posterior probability, provided the probability is greater than 0.95 and the resulting sequence will be at least 10 frames long. Frames may remain unclassified if they do not meet these criteria. Sequences are created both for training and testing data portions, such that two sets of sequences are generated for each data split (odd and even clips): one by the HMM trained on that split, and one by the HMM trained on the other split. 
Figure 59. Permutation results for standard-MVAR single-lag models, for each subject and phrase-type (GN = "Good news", ISTS = "I'm sorry to say"). Kernel density estimates show distributions of cross-validated correlations between predicted and actual trailing PCA scores: the dashed line indicates the empirical null distribution; the solid line indicates the true distribution. The dotted line indicates the one-tailed critical value for significance $\left(95^{\text {th }}\right.$ percentile of null distribution). The dashed-dotted line indicates the true mean. Annotated $p$-values indicate the proportion of scores in the null distribution greater than the true mean after correction for multiple comparisons.

Figure S10. Permutation results for standard-MVAR multi-lag models, for each subject and phrase-type (GN = "Good news", ISTS = "I'm sorry to say"). Other details as per Figure S9.

Figure S11. Standard-MVAR: example regression coefficients (even clips of "Good news" phrases) for (a) second, (b) third, and (c) fourth-order multi-lag models. Annotations indicate sections of matrix corresponding to each lag. Note that matrices have been transposed for display, such that trailing (outcome) components are displayed on rows and leading (predictor) components on columns. Note also that the first-order multi-lag models are not illustrated as they are equivalent to the $40 \mathrm{~ms}$ single-lag models (see Figure $3 a$ ).

Figure S12. Back-projected HMM state means for cross-validated datasets, for all subjects and phrase-classes (GN = "Good news", ISTS = "I'm sorry to say") and for selected models.

Figure S13. Permutation results for HMM-MVAR single-lag models, for each subject and phrase-type (GN = "Good news", ISTS = "I'm sorry to say"). Other details as per Figure S9.

Figure S14. Permutation results for HMM-MVAR multi-lag models, for each subject and phrase-type (GN = "Good news", ISTS = "I'm sorry to say"). Other details as per Figure S9.

\section{Supplementary Videos}

Video S1. Subject 1, "Good news": back-projected full-dataset HMM state means animation. State means are weighted by time courses of state posterior probabilities and then back projected to the image space. This produces an animation illustrating changes in behavioural states identified by the HMM over time.

Video S2. Subject 1, "I'm sorry to say": back-projected full-dataset HMM state means animation. Other details as per Video S1. 
Video S3. Subject 2, "Good news": back-projected full-dataset HMM state means animation. Other details as per Video S1.

Video S4. Subject 2, "I'm sorry to say": back-projected full-dataset HMM state means animation. Other details as per Video S1.

Video S5. Subject 3, "Good news": back-projected full-dataset HMM state means animation. Other details as per Video S1.

Video S6. Subject 3, "I'm sorry to say": back-projected full-dataset HMM state means animation. Other details as per Video S1. 